Digestive Endoscopy

\title{
Outcomes and timing of endoscopic retrograde cholangiopancreatography for acute biliary pancreatitis
}

\author{
Adrienn Halász ${ }^{a}$, Dániel Pécsi ${ }^{b}$, Nelli Farkas ${ }^{b, c}$, Ferenc Izbéki ${ }^{a}$, László Gajdán ${ }^{a}$, \\ Roland Fejes ${ }^{a}$, József Hamvas ${ }^{\mathrm{d}}$, Tamás Takács ${ }^{\mathrm{e}}$, Zoltán Szepes ${ }^{\mathrm{e}}$, László Czakó ${ }^{\mathrm{e}}$, \\ Áron Vincze ${ }^{\mathrm{f}}$, Szilárd Gódi ${ }^{\mathrm{f}}$, Andrea Szentesi ${ }^{\mathrm{b}, \mathrm{e}}$, Andrea Párniczky ${ }^{\mathrm{g}}$, Dóra Illés ${ }^{\mathrm{e}}$, \\ Balázs Kui ${ }^{e}$, Péter Varjú ${ }^{b}$, Katalin Márta ${ }^{b}$, Márta Varga ${ }^{\text {h }}$, János Novák ${ }^{i}$, Attila Szepes ${ }^{j}$, \\ Barnabás Bod ${ }^{\mathrm{k}}$, Miklós Ihász ${ }^{\mathrm{l}}$, Péter Hegyi ${ }^{\mathrm{b}, \mathrm{m}, \mathrm{n}}$, István $\mathrm{Hritz}^{\circ}$, \\ Bálint Erőss ${ }^{b, *}$, on behalf of the Hungarian Pancreatic Study Group
}

a Szent György Teaching Hospital of Fejér County, Székesfehérvár, Hungary

b Institute for Translational Medicine, Medical School, University of Pécs, Pécs, Hungary

${ }^{\mathrm{c}}$ Institute of Bioanalysis and Institute for Translational Medicine, Medical School, University of Pécs, Pécs, Hungary

${ }^{\mathrm{d}}$ Bajcsy-Zsilinszky Teaching Hospital of Semmelweis University, Budapest, Hungary

e First Department of Medicine, University of Szeged, Szeged, Hungary

${ }^{\mathrm{f}}$ First Department of Medicine, Medical School, University of Pécs, Pécs, Hungary

${ }^{g}$ Heim Pál National Institute for Pediatrics, Budapest, Hungary

h BMKK, Dr. Réthy Pál Hospital, Békéscsaba, Hungary

i BMKK, Pándy Kálmán Hospital, Gyula, Hungary

j Bács-Kiskun County University Teaching Hospital, Kecskemét, Hungary

${ }^{\mathrm{k}}$ Dr. Bugyi István Hospital of Csongrád County, Szentes, Hungary

${ }^{1}$ Markusovszky Teaching Hospital, Szombathely, Szombathely, Hungary

${ }^{m}$ First Department of Medicine, University of Pécs, Pécs, Hungary

${ }^{\mathrm{n}}$ MTA-SZTE Momentum Translational Gastroenterology Research Group, Szeged, Hungary

${ }^{\circ}$ First Department of Surgery, Center for Therapeutic Endoscopy, Semmelweis University, Budapest, Hungary

\section{A R T I C L E I N F O}

\section{Article history:}

Received 30 December 2018

Accepted 21 March 2019

Available online 25 April 2019

\section{Keywords:}

Cholangiopancreatography

Cholangitis

Complications

Endoscopic retrograde

Health care

Pancreatitis

Quality indicators

Registries

\begin{abstract}
A B S T R A C T
Background: Indication of endoscopic retrograde cholangiopancreatography (ERCP) in acute biliary pancreatitis (ABP) is challenging.

Aims: In this retrospective study, we analyzed real-world data to understand the ERCP practice in ABP in Hungarian centers.

Methods: Clinical data on ABP patients (2013-2015) were extracted from our large multicentric database. Outcomes, quality indicators and the role of early timing of ERCP ( $<24 \mathrm{~h}$ from admission) were analyzed. Results: There were 356 patients with ABP. ERCP was performed in 267 (75\%). Performance indicators of ERCP proved to be suboptimal with a biliary cannulation rate of $84 \%$. Successful vs unsuccessful cannulation of naïve papilla resulted in lower rates of local $[22.9 \%$ vs $40.9 \%,(P=0.012)]$ and systemic $[4.9 \%$ vs $13.6 \%$, $(P=0.042)]$ complications. Successful vs unsuccessful clearance resulted in lower rates of local complications [22.5\% vs $40.8 \%,(P=0.008)]$. Successful cannulation and drainage correlated with less severe course of $\mathrm{ABP}$ [3.6\% vs $15.9 \%,(\mathrm{P}=0.001)$ and $4.1 \%$ vs $12.2 \%,(\mathrm{P}=0.033)]$ respectively. A tendency of an increased rate of local complications was observed if ERCP was performed later [ $<24 \mathrm{~h}: 21.1 \%(35 / 166)$; between 24-48 h: 23.4\% (11/47); >48h: 37.2\% (16/43) $(\mathrm{P}=0.088)]$.

Conclusion: Optimization of ERCP indication in ABP patients is critical as suboptimal ERCP practices in $\mathrm{ABP}$ without definitive stone detection are associated with poorer clinical outcomes.
\end{abstract}

(c) 2019 Published by Elsevier Ltd on behalf of Editrice Gastroenterologica Italiana S.r.l.

\footnotetext{
* Corresponding author at: Institute for Translational Medicine, Medical School, University of Pécs, Szigeti str. 12, Pécs, 7624, Hungary.

E-mail addresses: ahalasz@mail.fmkorhaz.hu (A. Halász), daniel.pecsi1991@gmail.com (D. Pécsi), nelli.farkas@aok.pte.hu (N. Farkas), fizbeki@gmail.com (F. Izbéki), lgajdan@yahoo.com (L. Gajdán), rolldoc@vipmail.hu (R. Fejes), hamvas.jozsef@bajcsy.hu (J. Hamvas), takacs.tamas@med.u-szeged.hu (T. Takács), szepes.zoltan@med.u-szeged.hu (Z. Szepes), czako.laszlo@med.u-szeged.hu (L.Czakó),

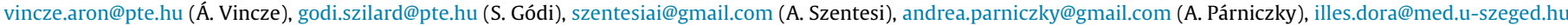

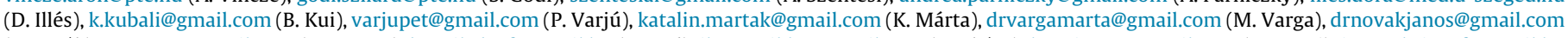

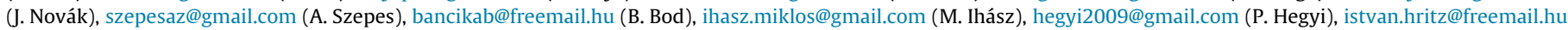
(I. Hritz), eross.balint@pte.hu (B. Erőss).
} 


\section{Core tip}

The endoscopic management of acute biliary pancreatitis is still a controversial topic. In this prospectively collected cohort of patients in Hungary, we found that successful cannulation and clearance at the first attempt are associated with better outcomes and suboptimal ERCP practices may have a negative impact on the outcomes of the disease.

\section{Introduction}

Acute pancreatitis (AP) is one of the most common diseases of the gastrointestinal tract requiring acute hospitalization; it is associated with significant morbidity and mortality worldwide with an increasing incidence of 5-100/100,000 cases per year [1]. Despite its importance, research on pancreatitis is continuously decreasing, suggesting that more attention should be paid to this disease [2].

One of the main etiological factors in the pathogenesis of AP is the obstruction of the ampulla of Vater by gallstones or sludge or by hypertrophy of papilla and bile reflux into the pancreatic duct, contributing to $35-60 \%$ of all AP cases. This subtype of AP is termed acute biliary or gallstone pancreatitis (ABP) [3]. Management of $\mathrm{ABP}$ requires two treatment strategies in most cases. The general, conservative medical treatment consists of appropriate fluid resuscitation (preferably lactated Ringer's solution) [4], pain management, and enteral nutrition in some cases [5]. The use of the interventional strategy in ABP to achieve biliary decompression is still uncertain. In cases of concomitant acute cholangitis, the need for urgent $(<24 \mathrm{~h})$ endoscopic retrograde cholangiopancreatography (ERCP) is recommended. There is also a clear indication of ERCP in cases of obstruction where biliary drainage must be properly resolved in a short period of time [1,6-8]. On the other hand, without apparent signs of cholangitis or obstruction (manifest systemic inflammation, biliary stones or dilatation on imaging, and jaundice or abnormal liver function test), the indication of ERCP in the setting of ABP is still debated because of the lack of available evidence $[1,6]$. The data provided by meta-analyses of randomized controlled trials (RCTs), where the most recent ones analyzed 10 and 11 RCTs, demonstrated a significant decrease in complications, hospital stay, and cost in patients with ABP managed with early ERCP (within $72 \mathrm{~h}$ ) compared to conservative management $[9,10]$. An ongoing RCT is organized by the Dutch Pancreatitis Study Group on this still controversial topic; the APEC trial is set to determine the role of early ERCP with biliary sphincterotomy in ABP without cholangitis [11]. Despite the studies noted above, optimal comprehensive management of ABP is still lacking clear evidence.

The Hungarian Pancreatic Study Group (HPSG) was created in 2011 to improve patient care in pancreatic diseases and within a short period; this organization has produced several pancreatic registries, trials and established guidelines [12-16]. One of its registries, concerning Acute Pancreatitis, has recorded data on AP cases from all participating centers throughout Hungary and now in more than a dozen countries [17].

This study aims to determine the role of ERCP and sphincterotomy and stone clearance in ABP and to provide an overview of the general use of ERCP in Hungary in this disease.

\section{Materials and methods}

\subsection{Inclusion criteria}

All patients with AP were enrolled, and their data were prospectively collected in the HPSG AP Registry, which has been approved by the Scientific and Research Ethics Committee of the Medical Research Council (TUKEB-22254-1/2012/EKU). All patients were informed about the data collection and signed the informed consent forms. An AP diagnosis was made according to the recommendations in the IAP/APA guidelines, with at least two of the following three criteria met: abdominal pain, pancreatic enzyme exceeding more than three times the upper normal level, and features of pancreatitis on imaging.

In this cohort study, we selected patients who fit the criteria previously laid down by the Dutch Pancreatic Study Group, which were used to determine biliary origin: (a) gallstones and/or sludge diagnosed on transabdominal ultrasound or computed tomography (CT) or (b) dilated CBD on ultrasound or CT (diameter: $>8 \mathrm{~mm}$ for age $\leq 75$ years and diameter: $>10 \mathrm{~mm}$ for age $>75$ years) or (c) two of the following three laboratory abnormalities: (1) serum bilirubin level $>1.3 \mathrm{mg} / \mathrm{dL}$ [ $>40 \mu \mathrm{mol} / \mathrm{L}]$; (2) alanine aminotransferase (ALT) level $>100 \mathrm{U} / \mathrm{L}$ with an ALAT level greater than the aspartate aminotransferase level; and (3) alkaline phosphatase level >195 U/L with a gamma-glutamyltransferase level $>45 \mathrm{U} / \mathrm{L}$. Other causes of AP, such as alcohol, hypertriglyceridemia, diet, drug-induced, trauma, viral infection, post-ERCP, and idiopathic AP had to be absent (Supplementary Table 1) [18].

691 patients with AP were enrolled in the AP registry between January 2013 and August 2015 from 14 centers. The manuscript was prepared in accordance with the STROBE statement [19].

\subsection{Exclusion criteria}

Patients under the age of 18 years and those with non-biliary pancreatitis were excluded from the analysis.

\subsection{Data extraction}

Data on demographics (sex and age), etiology, severity, and mortality of AP were extracted for all subjects with AP, and a descriptive statistical analysis was performed. The severity of AP was classified according to the revised Atlanta classification as mild, moderately severe, and severe [20]. Main outcomes were the severity of pancreatitis, local (peripancreatic fluid, pseudocyst, necrosis of pancreas on imaging, diabetes mellitus, and abdominal compartment syndrome) and systemic (transient or persistent organ failure based on the modified Marshall scoring system for organ dysfunction) complications, mortality, and length of hospital stay.

Detailed demographics, including body mass index, comorbidities, and data on outcomes for ERCP, were collected on patients with ABP, such as indication of ERCP, successful cannulation rate, management of CBD stones by sphincterotomy and duct clearance, biliary and pancreatic stenting, anatomy of the papilla (naïve/not naïve), and complication rates (bleeding and perforation). Timing of ERCP was calculated from admission. The outcomes for ABP (severity of pancreatitis, local and systemic complications, mortality, and length of hospital stay) were analyzed in relation to the timing of ERCP. No follow-up was carried out after hospital discharge.

\subsection{Statistical analysis}

Continuous measures are summarized and presented as means and standard deviations (SD) or as median and interquartile ranges (IQR). Categorical data are presented as observed and as percentages. To determine differences between continuous parameters, depending on the distribution of the data, we used the independent Student's t-test or the Mann-Whitney U test for two groups and one-way ANOVA with the Bonferroni post-hoc test or Kruskal-Wallis test in comparing more than two groups. We used the Chi-square test or Fisher's exact test to analyze the rela- 
Table 1

General characteristics of the acute biliary pancreatitis cohort.

\begin{tabular}{|c|c|c|c|c|}
\hline & All $[\mathrm{n}(\%)]$ & Women [n (\%)] & Men $[\mathrm{n}(\%)]$ & P-value \\
\hline Gender & 356 & $204(57.3 \%)$ & $152(42.7 \%)$ & - \\
\hline Age & $61.65 \pm 17.32$ & $61.3 \pm 18.1$ & $62.1 \pm 16.1$ & NS \\
\hline Prior cholecystectomy & $35(9.4 \%)$ & $28(13.9 \%)$ & $7(4.6 \%)$ & 0.004 \\
\hline Previously documented pancreatitis & $42(11.8 \%)$ & $26(12.7 \%)$ & $16(10.5 \%)$ & NS \\
\hline Diabetes mellitus & $59(16.6 \%)$ & $26(12.7 \%)$ & $33(21.7 \%)$ & 0.023 \\
\hline Body mass index (available for 160 women and 123 men) & $28.31 \pm 6.1$ & $28.24 \pm 6.03$ & $28.40 \pm 6.22$ & NS \\
\hline At least 2 co-morbidities & $155(43.5 \%)$ & $85 / 204(41.7 \%)$ & $70 / 152(46.1 \%)$ & NS \\
\hline
\end{tabular}

tions between the factors under examination. All analyses were performed with SPSS 24 statistical software (IBM Corporation).

\section{Results}

\subsection{General characteristics of the AP cohort}

Biliary etiology was found in 356 (51.5\%) patients, and 335 (48.5\%) patients had other etiological factors (alcohol, hypertriglyceridemia, diet, drug-induced, trauma, viral infection, post-ERCP, and idiopathic AP). Among the subjects with ABP, there were more women, and they were older than patients with a different etiology [204/356 (57.3\%) vs 106/335 (31.6\%) (P<0.001)] and mean age $[61.5 \pm 17.32$ vs $51.47 \pm 15.73$ years $(\mathrm{P}<0.001)]$. The course of pancreatitis with biliary etiology was milder in contrast to non-ABP disease [mild ABP: 248/356 (69.7\%) vs non-ABP: 183/335 (54.6\%); moderately severe ABP: 86/356 (24.2\%) vs nonABP: $121 / 335$ (36.1\%); severe ABP: $22 / 356$ (6.2\%) vs non-ABP: $31 / 335(9.2 \%)(\mathrm{P}<0.001)]$. There was no difference in mortality between the two groups [ABP: $8 / 336$ (2.4\%) vs non-ABP: 13/322 (4.0\%) $(\mathrm{P}=0.242)]$.

\subsection{Characteristics of the ABP cohort}

A pancreatitis diagnosis was based on upper abdominal pain and elevated pancreatic enzymes in $327 / 356(91.8 \%)$ of the patients.

ABP occurs more commonly in women [204/356 (57.3\%) female vs $152 / 356(42.7 \%)$ male]. In almost $10 \%$ of the cases, ABP developed after a cholecystectomy [35/356 (9.4\%)] and more frequently in women [28/204 (13.9\%) vs 7/152 (4.6\%) ( $\mathrm{P}=0.004)]$.

Diabetes mellitus as a co-morbidity was found in $16.6 \%(59 / 356)$ of the patients, significantly more often in men [26/204 (12.7\%) vs 33/152 (21.7\%) $(\mathrm{P}=0.023)]$.

Age, a previously documented episode of pancreatitis, body mass index (BMI), and co-morbidity were not different between the two sexes. A considerable number of ABP patients had more than two co-morbidities [43.5\% (155/356)] (Table 1).

\subsection{Indications for ERCP}

Out of the 356 patients, 267 underwent ERCP (75.0\%) for suspected cholangitis or cholestasis without cholangitis based on raised inflammatory markers with dilated biliary ducts and raised liver function tests. 89 patients in total did not undergo ERCP although it would have been indicated in 50 cases of suspected cholangitis (56.2\%) ERCP was not performed in these cases due to an improving clinical picture, lack of consent from the patient, or rapid deterioration of multi-organ failure.

Endoscopic ultrasonography (EUS) was performed in only five patients, with bile duct stones being identified in two cases. MRCP was carried out in one patient, in which clear bile ducts were reported.

\subsection{Quality indicators and findings of ERCP}

The key performance indicators for ERCP met the criteria set out in the American Society of Gastrointestinal Endoscopy (ASGE) guidelines [21]. Successful biliary cannulation was achieved in 233 subjects with naïve papilla (90.7\%), but the successful cannulation rate was $84.0 \%$ (216 procedures) at first attempt. In 80 subjects, extractions of stones smaller than $1 \mathrm{~cm}$ were successful in $93.7 \%$ of the cases. Stent implantation below the bifurcation was successfully carried out in all cases after successful deep biliary cannulation (33/33). Perforation occurred in $1 / 267$ ( $0.4 \%$ ) of the cases. Clinically significant bleeding requiring blood transfusion developed in 3/267 $(1.2 \%)$ of the patients.

Common bile duct (CBD) stones, sludge, and/or dilation of the bile ducts were reported in 97 (36.3\%), 91 (34.1\%), and $124(46.4 \%)$ cases, respectively. Spontaneous passage of a bile duct stone was suspected in 19.5\% (52/267) of the patients during ERCP. In 30 cases (11.2\%), no biliary pathology was found by ERCP. Endoscopic ultrasound was only carried out in five cases because of limited access at the time of data collection.

315 ERCPs were performed in 267 patients until completion or abandoning the intervention or treatment. 43 patients had two ERCPs, and five had three procedures.

\subsection{Outcomes for $A B P$ in relation to success rates of ERCP}

Data on cannulation success rate and clearance of the bile ducts were available in all cases. The success rate for bile duct cannulation in all patients was $83.5 \%$ (223/267) during the first ERCP, and any further endoscopic attempts resulted in a higher rate of success [90.6\% (242/267)]. Successful cannulation was achieved in $84.0 \%$ (216/257) of patients with naïve papilla, and clearance of the bile duct was successful in $71.5 \%(191 / 267)$ at the first ERCP attempt. Endoscopic biliary sphincterotomy was done in 86.5\% (231/267) of the ERCPs, whereas pancreatic sphincterotomy was only performed in $1.12 \%(3 / 267)$ of the cases. Biliary stents were placed in $12.36 \%$ (33/267) and pancreatic stents in 16.85\% (45/267) of the cases. Successful cannulation was associated with significantly lower rates of local and systemic complications. Successful clearance was linked to lower rates of local complications. Successful cannulation and clearance both correlated with a less severe course of ABP and shorter hospitalization (Tables 2 and 3 ).

Complete failure of clearance and decompression of the bile ducts were related to higher frequency of local complications and a more severe course of ABP and longer hospital stay (Table 4).

\subsection{Outcomes for $A B P$ in relation to the timing of $E R C P$}

ERCP was performed in $75 \%$ (267/356) of the cases, the majority of them during the first $24 \mathrm{~h}$ after admission.

Data on the timing of ERCP were available in 256 (95.9\%) cases. ERCP was performed on $64.8 \%(166 / 256)$ of the patients within $24 \mathrm{~h}$ after admission, in $18.4 \%$ (47/256) of them between 24 and $48 \mathrm{~h}$ after admission, and in $16.8 \%(43 / 256)$ cases later than $48 \mathrm{~h}$ after admission. A tendency of an increased rate of local complications 
Table 2

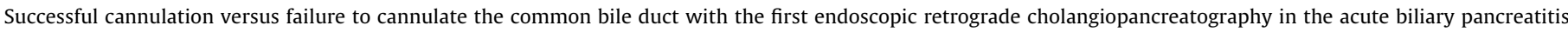
cohort.

\begin{tabular}{|c|c|c|c|}
\hline & Successful bile duct cannulation with 1st ERCP [n (\%)] & Failure to cannulate the bile duct with 1 st ERCP [n (\%)] & P-value \\
\hline Total $(n=267)$ & $223(83.5 \%)$ & $44(16.5 \%)$ & - \\
\hline Rates of severe disease & $8(3.6 \%)$ & $7(15.9 \%)$ & 0.001 \\
\hline Local complications & $51(22.9 \%)$ & $18(40.9 \%)$ & 0.012 \\
\hline Systemic complications & $11(4.9 \%)$ & $6(13.6 \%)$ & 0.042 \\
\hline Mortality & $4(1.8 \%)$ & $1(2.3 \%)$ & NS \\
\hline Hospital stay, median (IQR) & $9(6-13)$ & $14(8-21.5)$ & 0.00021 \\
\hline
\end{tabular}

Table 3

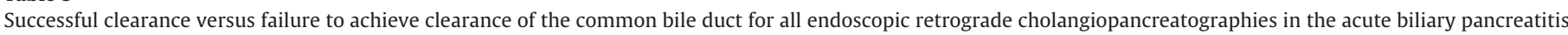
cohort.

\begin{tabular}{|c|c|c|c|}
\hline & Successful bile duct clearance [n (\%)] & Failure of clearance the bile duct [n (\%)] & P-value \\
\hline Total $(n=267)$ & $218(81.6 \%)$ & $49(18.3 \%)$ & - \\
\hline Rates of severe disease & $9(4.1 \%)$ & $6(12.2 \%)$ & 0.033 \\
\hline Local complications & $49(22.5 \%)$ & $20(40.8 \%)$ & 0.008 \\
\hline Systemic complications & $13(6.0 \%)$ & $4(8.2 \%)$ & NS \\
\hline Mortality & $4(1.8 \%)$ & $1(2.0 \%)$ & NS \\
\hline Hospital stay, median (IQR) & $9(6-13)$ & $11(7-21)$ & 0.021 \\
\hline
\end{tabular}

\section{Table 4}

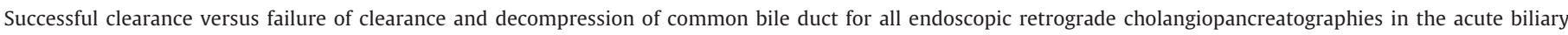
pancreatitis cohort.

\begin{tabular}{|c|c|c|c|}
\hline & Successful clearance of bile duct [n (\%)] & Unsuccessful clearance and decompression of bile duct [n (\%)] & P-value \\
\hline Total & $218 / 267(81.6 \%)$ & $32 / 250(12.8 \%)$ & - \\
\hline Rates of severe disease & $9(4.1 \%)$ & $6(18.7 \%)$ & 0.001 \\
\hline Local complications & $49(22.5 \%)$ & $16(50.0 \%)$ & 0.001 \\
\hline Systemic complications & $13(6.0 \%)$ & $4(12.5 \%)$ & NS \\
\hline Mortality & $4(1.8 \%)$ & $1(3.1 \%)$ & NS \\
\hline Hospital stay median (IQR) & $9(6-13)$ & $16(8.5-24.5)$ & 0.001 \\
\hline
\end{tabular}

Table 5

Outcomes for acute biliary pancreatitis in relation to the timing of endoscopic retrograde cholangiopancreatography in all patients with acute biliary pancreatitis.

\begin{tabular}{|c|c|c|c|c|}
\hline & $<24 \mathrm{~h}[\mathrm{n}(\%)]$ & $24-48 \mathrm{~h}[\mathrm{n}(\%)]$ & $>48 \mathrm{~h}[\mathrm{n}(\%)]$ & P-value \\
\hline Total $(n=256)$ & $166(64.8 \%)$ & $47(18.36 \%)$ & $43(16.80 \%)$ & - \\
\hline Rates of severe disease & $5(3 \%)$ & $3(6.4 \%)$ & $3(7.0 \%)$ & NS \\
\hline Local complications & $35(21.1 \%)$ & $11(23.4 \%)$ & $16(37.2 \%)$ & 0.088 \\
\hline Systemic complications & $7(4.2 \%)$ & $4(8.5 \%)$ & $4(9.3 \%)$ & $\mathrm{N} / \mathrm{A}^{*}$ \\
\hline Mortality & $2(1.2 \%)$ & $1(2.1 \%)$ & $0(0 \%)$ & $\mathrm{N} / \mathrm{A}^{*}$ \\
\hline Hospital stay, median (IQR) & $8(6-12)$ & $10(5.5-15)$ & $13(9.5-21)$ & $<0.001$ \\
\hline
\end{tabular}

${ }^{*}$ Statistical analysis was not carried out in cases of systemic complications, mortality, and rates of severe disease due to low numbers of subjects.

was observed if ERCP was performed later [ERCP in $24 \mathrm{~h}: 21.1 \%$ (35/166); between 24 and 48 h: $23.4 \%$ (11/47); after 48 h: $37.2 \%$ $(16 / 43)(P=0.088)]$.

We note that only $1.1 \%$ (7/267) of the endoscopic interventions described signs of purulent cholangitis.

The length of hospitalization was significantly longer in all patients if ERCP was delayed (Table 5).

\subsection{Other relevant findings}

There was no statistically significant difference in the outcomes for ABP between the patients treated with or without ERCP. (Supplementary Table 2 ).

Use of antibiotics was a common practice. 87.6\% (312/356) of all the patients received antibiotics, for which the indication was suspected cholangitis in 85.3\% (266/312). They were administered to treat infections outside the biliary tree, such as pneumonia and urinary tract infections, in $11.9 \%(37 / 312)$ of the cases. The first choice among antibiotics for cholangitis was a combination of a cephalosporin and metronidazole.

\section{Discussion}

Prospectively collected, real-world data were analyzed in this multicenter study, and considerable coverage of Hungarian acute biliary pancreatitis cases is presented. Data shown above depict current management strategies used in Hungary.

Although a clear-cut diagnosis of definite cholangitis would have been desirable in our analysis, currently there is no validated definition of cholangitis in the setting of ABP. Simple AP can result in a transient and self-resolving biliary obstruction with deranged liver function tests and dilated biliary tree, which can mimic cholangitis with the raised inflammatory markers driven by pancreatitis. Thus the use of the definition of definite cholangitis as termed by the Tokyo criteria had to be avoided [18].

As described in other studies, we found that patients with ABP are older, and there are more women among them compared to AP of other etiologies [3]. In the Hungarian cohort, $A B P$ tended to have a less severe natural course, but the mortality was the same as in other etiologies, as reported in a large study [2].

Previous cholecystectomy was relatively common and more so in women. This could be explained by the fact that biliary stone disease is more common in females and that stone disease of the 
gallbladder increases the risk of $\mathrm{ABP}$, most likely even after a previous cholecystectomy. A previously documented episode of AP occurred in more than $10 \%$ of the patients. Although data on the etiology of the previous attack was not available, we believe that the majority of the cases were likely driven by gallstone disease, similar to data reported by Godi et al. [12]. Diabetes is a known risk factor for AP, and significantly more men had diabetes in the Hungarian cohort, which was reported in AP with all etiologies [15].

ERCP was performed in $75 \%$ of the patients presenting with ABP. To our best knowledge, there are no previous cohort studies where the rate of ERCP was published and analyzed. We found that our ERCP practice in ABP is in line with the current guidelines; however, we must highlight that very limited access to urgent endoscopic ultrasound (EUS) and magnetic resonance cholangiopancreatography (MRCP) resulted in a number of avoidable ERCPs. At the same time, a small proportion of the patients with suspected cholangitis were not amenable to ERCP. In summary, we believe that ERCP for $\mathrm{ABP}$ will be reduced as access to EUS and MRCP improves. In most cases, the indication was suspected cholangitis, and these are the patients who could have benefited most from additional diagnostic imaging $[16,19,22]$.

Our results clearly demonstrated that the lack of access to additional diagnostic tools (EUS or MRCP) resulted in a high number of unnecessary and avoidable ERCPs, and this clinical practice needs to be improved.

Some of the key performance indicators describing the ERCP practices in this large cohort across many centers described suboptimal ERCP practices. Most importantly a success rate of $84 \%$ at first attempt (216 patients), which is below the quality benchmark of $>90 \%$ recommended by ASGE. This may well be driven by the fact that some of the ERCPs were performed in low volume centers. It also reminds us that, if indicated, high-quality ERCP with maximal pancreas protection and high competence of alternative biliary access techniques should be mandatory.

One of our main findings is that failed cannulation and bile duct clearance are associated with a higher incidence of local complications and severity of ABP. This result can be interpreted in two ways. Firstly, successful clearance and decompression of the bile ducts can result in a quicker resolution of pancreatitis and less progression leading to complications. Secondly, it may be explained by the difficult access to the bile ducts in already complicated AP, driven by difficult intubation of the duodenum, poor visualization of the papilla, limited maneuverability of the duodenoscope, and challenging cannulation of the edematous papilla. ERCPs are therefore done for the indication of acute biliary pancreatitis classified as grade 3 difficulty on the modified Schutz grade, on a scale of 1-4, where 4 is the most difficult [23].

In this situation, high success rates can only be expected of competent, highly skilled endoscopists with substantial case numbers. This is how we explain the slightly suboptimal quality indicators (ASGE guideline) of ERCP in this cohort [19]. Cannulation of naïve papilla was successful at first attempt in $84.1 \%$ of all ERCPs (desired: $90 \%$ ), perforation occurred in $0.4 \%$ (desired: $\leq 0.2 \%$ ), and bleeding requiring transfusion resulted in $1.2 \%$ (desired: $\leq 1 \%$ ). We note that this analysis contained data from 267 patients, hence the two latter measures. Quality indicators of stone extraction and stenting of obstructions below the level of bifurcation met the criteria for the guidelines.

We did not find a significant decrease in the rate of local complications and hospital stay in the cohort when ERCP was performed within two days. Evidence suggests that early ERCP in ABP with cholangitis is indicated $[1,6-8]$, but our findings could not reinforce these previous data. In patients with a clear-cut diagnosis of acute cholangitis, ERCP should be considered as soon as possible to provide a better outcome [24].
Just like the need for ERCP, the high rate of antibiotic use reported in our study (87.6\%) could possibly be reduced by better access to EUS and MRCP in the case of suspected cholangitis. However, cholangitis is one of the most feared sources of abdominal infection and can lead to sepsis, multiple organ failure, and death. Therefore, any strategy to delay or withhold antibiotics in the context of suspected cholangitis should be carefully assessed. Educational activities should be organized and materials disseminated to ensure strict adherence to international guidelines $[1,6]$.

\subsection{Strengths and weaknesses of the study}

This cohort represents a general, diverse, multicenter (not only tertiary centers participated), acute biliary pancreatitis sample. For this reason, broader, more generalizable conclusions could be drawn. Limitations of this study are the relatively low case numbers in subgroups and the retrospective design with post-hoc question raising, which is susceptible to biases, thus limiting the conclusions considerably. Lastly, a large number of participating ERCP units without a structured approach to the timing of ERCP procedures in ABP limits the statistical conclusions on associations with the outcomes of pancreatitis.

\section{Conclusion}

The indication and benefit of ERCP in patients with ABP but without a clear-cut diagnosis, cholangitis remains a contentious issue. We recommend that the non-invasive diagnostic approach should be maximized to select the most suitable subgroup of these patients. As ERCP is difficult in ABP, quality indicators must be closely monitored, and procedures should be performed by experts in high-volume centers as suboptimal ERCP practices are likely to be associated with poorer outcomes of the acute biliary pancreatitis.

\section{Funding}

This study was supported by Project Grants (KH125678 and K116634 to PH, K120335 to TT), the Economic Development and Innovation Operative Programme Grant (GINOP 2.3.2-15-372 2016-00048 to PH) and Human Resources Development Operational Programme Grant (EFOP-3.6.2-16-2017-00006 to PH) from the National Research, Development and Innovation Office, by a Momentum Grant from the Hungarian Academy of Sciences (LP2014-10/2014 to PH), by the János Bolyai Research Scholarship of the Hungarian Academy of Sciences (to AP) and the ÚNKP18-4 new national excellence program of the Ministry of Human Capacities (to AP). Funders had no role in study design, data collection, analysis, interpretation of the findings, and preparation of the manuscript.

\section{Institutional review board statement}

This study was approved by the Hungarian Scientific and Research Committee of Medical Research Council (MRC). All patients with acute pancreatitis were enrolled, and their data were prospectively collected in the Hungarian Pancreatic Study Group (HPSG) AP Registry, which has been approved by the Scientific and Research Ethics Committee of the Medical Research Council (TUKEB-22254-1/2012/EKU). All patients were informed about the data collection and signed the informed consent forms.

\section{Conflict of interest}

Adrienn Halász MD, Dániel Pécsi MD, Nelli Farkas MSc PhD, Ferenc Izbéki MD PhD, László Gajdán MD, Roland Fejes MD, József Hamvas MD, Tamás Takács MD PhD DSc, Zoltán Szepes MD PhD, László Czakó MD PhD DSc, Áron Vincze MD PhD DSc, Szilárd Gódi MD, 
Andrea Szentesi MSc PhD, Andrea Párniczky MD PhD, Dóra Illés MD, Balázs Kui MD PhD, Péter Varjú MD, Katalin Márta MD, Márta Varga MD, János Novák MD, Attila Szepes MD PhD, Barnabás Bod MD, Miklós Ihász MD, Péter Hegyi MD PhD DSc, István Hritz MD PhD and Bálint Erőss MD have participated in (a) conception and design, or analysis and interpretation of the data; (b) drafting the article or revising it critically for important intellectual content; and (c) approval of the final version. The manuscript has not been submitted to, nor is under review at, another journal or other publishing venue. The authors have no affiliation with any organization with a direct or indirect financial interest in the subject matter discussed in the manuscript.

\section{Appendix A. Supplementary data}

Supplementary material related to this article can be found, in the online version, at doi:https://doi.org/10.1016/j.dld.2019.03. 018.

\section{References}

[1] Crockett SD, Wani S, Gardner TB, Falck-Ytter Y, Barkun AN. American Gastroenterological Association Institute guideline on initial management of acute pancreatitis. Gastroenterology 2018;154:1096-101.

[2] Szentesi A, Toth E, Balint E, Fanczal J, Madácsy T, Laczkó D, et al. Analysis of research activity in gastroenterology: pancreatitis is in real danger. PLoS One 2016;11:e0165244.

[3] van Geenen EJ, van der Peet DL, Bhagirath P, Mulder CJ, Bruno MJ. Etiology and diagnosis of acute biliary pancreatitis. Nat Rev Gastroenterol Hepatol 2010; 7:495-502.

[4] de-Madaria E, Herrera-Marante I, Gonzalez-Camacho V, Bonjoch L, QuesadaVázquez N, Almenta-Saavedra I, et al. Fluid resuscitation with lactated Ringer's solution vs normal saline in acute pancreatitis: A triple-blind, randomized, controlled trial. United European Gastroenterol J 2018;6:63-72.

[5] Vaughn VM, Shuster D, Rogers MAM, Mann J, Conte ML, Saint S, et al. Early versus delayed feeding in patients with acute pancreatitis: a systematic review. Ann Intern Med 2017;166:883-92.

[6] IAP/APA evidence-based guidelines for the management of acute pancreatitis. Pancreatology 2013;13:e1-15.

[7] Yokoe M, Takada T, Mayumi T, Yoshida M, Isaji S, Wada K, et al. Japanese guidelines for the management of acute pancreatitis: Japanese Guidelines 2015. J Hepatobiliary Pancreat Sci 2015;22:405-32.

[8] Miura F, Okamoto K, Takada T, Strasberg SM, Asbun HJ, Pitt HA, et al. Tokyo Guidelines 2018: initial management of acute biliary infection and flowchart for acute cholangitis. J Hepatobiliary Pancreat Sci 2018;25:31-40.

[9] Coutinho LMA, Bernardo WM, Rocha RS, Marinho FR, Delgado A, Moura ETH, et al. Early endoscopic retrograde cholangiopancreatography versus conserva- tive treatment in patients with acute biliary pancreatitis: systematic review and meta-analysis of randomized controlled trials. Pancreas 2018;47:444-53.

[10] Burstow MJ, Yunus RM, Hossain MB, Khan S, Memon B, Memon MA Meta-analysis of early endoscopic retrograde cholangiopancreatography (ERCP) +/- endoscopic sphincterotomy (ES) versus conservative management for gallstone pancreatitis (GSP). Surg Laparosc Endosc Percutan Tech 2015;25:185-203.

[11] Schepers NJ, Bakker OJ, Besselink MG, Bollen TL, Dijkgraaf MG, van Eijck CH, et al. Early biliary decompression versus conservative treatment in acute biliary pancreatitis (APEC trial): study protocol for a randomized controlled trial. Trials 2016;17:5.

[12] Godi S, Eross B, Gyomber Z, Szentesi A, Farkas N, Parniczky A, et al. Centralized care for acute pancreatitis significantly improves outcomes. J Gastrointestin Liver Dis 2018;27:151-7.

[13] Szucs A, Marjai T, Szentesi A, Farkas N, Parniczky A, Nagy G, et al. Chronic pancreatitis: multicentre prospective data collection and analysis by the Hungarian Pancreatic Study Group. PLoS One 2017;12:e0171420.

[14] Marta K, Szabo AN, Pecsi D, Varju P, Bajor J, Godi S, et al. High versus low energy administration in the early phase of acute pancreatitis (GOULASH trial): protocol of a multicentre randomised double-blind clinical trial. BMJ Open 2017;7:e015874.

[15] Parniczky A, Abu-El-Haija M, Husain S, Lowe M, Oracz G, Sahin-Tóth M, et al. EPC/HPSG evidence-based guidelines for the management of pediatric pancreatitis. Pancreatology 2018;18:146-60.

[16] Lakatos G, Balazs A, Kui B, Godi S, Szucs A, Szentesi A, et al. Pancreatic cancer: multicenter prospective data collection and analysis by the Hungarian Pancreatic Study Group. J Gastrointestin Liver Dis 2016;25:219-25.

[17] Parniczky A, Kui B, Szentesi A, Balazs A, Szucs A, Mosztbacher D, et al. Prospective, multicentre, nationwide clinical data from 600 cases of acute pancreatitis. PLoS One 2016;11:e0165309.

[18] van Santvoort HC, Besselink MG, de Vries AC, Boermeester MA, Fischer K, Bollen TL, et al. Early endoscopic retrograde cholangiopancreatography in predicted severe acute biliary pancreatitis: a prospective multicenter study. Ann Surg 2009;250:68-75.

[19] von Elm E, Altman DG, Egger M, Pocock SJ, Gotzsche PC, Vandenbroucke JP. Strengthening the Reporting of Observational Studies in Epidemiology (STROBE) statement: guidelines for reporting observational studies. BMJ 2007;335:806-8.

[20] Banks PA, Bollen TL, Dervenis C, Gooszen HG, Johnson CD, Sarr MG, et al. Classification of acute pancreatitis-2012: revision of the Atlanta classification and definitions by international consensus. Gut 2013;62:102-11.

[21] Baron TH, Petersen BT, Mergener K, Chak A, Cohen J, Deal SE, et al. Quality indicators for endoscopic retrograde cholangiopancreatography. Am J Gastroenterol 2006;101:892-7.

[22] Liu CL, Fan ST, Lo CM, Tso WK, Wong Y, Poon RT, et al. Comparison of early endoscopic ultrasonography and endoscopic retrograde cholangiopancreatography in the management of acute biliary pancreatitis: a prospective randomized study. Clin Gastroenterol Hepatol 2005;3:1238-44.

[23] Cotton PB, Eisen G, Romagnuolo J, Vargo J, Baron T, Tarnasky P, et al. Grading the complexity of endoscopic procedures: results of an ASGE working party. Gastrointest Endosc 2011;73:868-74.

[24] Parikh MP, Wadhwa V, Thota PN, Lopez R, Sanaka MR. Outcomes associated with timing of ERCP in acute cholangitis secondary to choledocholithiasis. J Clin Gastroenterol 2018;52(10):e97-102. 